\title{
Comparison of the Imaging and Pathological Features of Telangiectatic Osteosarcoma and Aneurysmal Bone Cyst of Long Tubular Bones
}

\author{
Yun-Heng Shi ${ }^{1}$, Shu-Man $\operatorname{Han}^{1}$, Tao Sun ${ }^{1}$, Bao-Hai Yu ${ }^{1}$, Hui-Zhao $\mathrm{Wu}^{1}$, Wen-Juan $\mathrm{Wu}^{1,}{ }^{\text {, }}$ and Bu-Lang \\ $\mathrm{GaO}^{1}$ \\ ${ }^{1}$ Department of Radiology, The Third Hospital of Hebei Medical University, Shijiazhuang, China \\ "Corresponding author: Department of Radiology, The Third Hospital of Hebei Medical University, 139 Ziqiang Road, Shijiazhuang, China. Tel: +86-18533112719, Email: \\ wenjwu@163.com
}

Received 2019 May 30; Revised 2020 March 07; Accepted 2020 March 18.

\begin{abstract}
Background: The clinical, imaging and pathological features of telangiectatic osteosarcoma (TOS) in long bones are helpful for differentiation from aneurysmal bone cysts (ABC).

Objectives: To investigate the clinical, imaging and pathological features of TOS in comparison with ABC in long bones.

Patients and Methods: Seventeen patients with TOS and 17 patients with ABC in our hospital were analyzed in the clinical, imaging and pathological features for proper diagnosis.

Results: For plain radiographs in 17 TOS cases, a soft tissue mass was detected in 14 cases, lytic bone destruction in 13, ill-defined border in 12, periosteal reaction in eight, formation of the Codman triangle in five, pathological fracture in three, and calcification in one. In 17 cases with ABC, an ill-defined border was found in fifteen cases, lytic and expansile destruction in eleven, thinned sclerotic rim in twelve, eccentrically location in ten, coarse septations in eight, a soft tissue mass in three, pathological fracture in three, periosteal reaction in two, and ill-defined border in two cases. In CT imaging of TOS, multiple cysts were in twelve cases, and fluidfluid levels in three. In ABC, multiple cysts were in eleven cases while fluid-fluid levels in four. In MRI presentations of TOS, low and medium signal was present in the T1-weighted imaging (T1WI), whereas mixed (9/12) or high signal (3/12) was presented in the T2 WI with inhomogeneous signal. Four cases had multiple cysts and fluid-fluid levels in the bone destruction and soft tissues. The cysts had long T1 and long T2 signal with isointense signal in the septations (5/12). Fat inhibition sequence showed clear cystic changes. In $\mathrm{ABC}$, low complete or incomplete signal was demonstrated in all lesions with a higher incidence of fluid-fluid levels (8/11). The lesion was of low and medium inhomogeneous signal in the T1 WI but mixed signal in T2 WI (6/11) including hyperintense signal in the upper part but mixed signal in the lower part in four cases or hyperintense signal (1/11) in T2 WI. The histopathological features of TOS were bleeding inside the lesion, highly atypia tumor cells of malignancy in the septa and tumorous bone tissue. The septa of $\mathrm{ABC}$ were composed of proliferative fibroblasts with dispersed polykaryocytes, reactive woven bone but no atypical cells.

Conclusion: The clinical, imaging and pathological characteristics should be combined to make a correct differential diagnosis between telangiectatic osteosarcoma and aneurysmal bone cyst.
\end{abstract}

Keywords: Telangiectatic Osteosarcoma, Aneurysmal Bone Cyst, Plain Radiography, Magnetic Resonance Imaging, Computed Tomography

\section{Background}

Osteosarcomas are the commonest primary malignant tumor of the bone at the metaphyses in children and adolescents. Osteosarcomas are divided into conventional, parosteal, intracortical, intramedullary, small-cell, giant cell-rich and telangiectatic osteosarcomas (TOS). As a rare subtype representing $2 \%-12 \%$ of all cases of osteosarcoma, TOS has the following characteristics of multiple, aneurysmally-dilated, blood-filled cavities with high-grade sarcomatous cells at the peripheral rim and within the septa (1-4). Similar to conventional osteosarcoma, the metaphyses of long tubular bones are common locations for this tumor, with the most frequent location of the femur followed by humerus and tibia. Identification of the unique histopathological and radiological characteristics of TOS is crucial for correct diagnosis. Nonetheless, TOS can be readily misdiagnosed as a benign tumor, especially aneurysmal bone cyst (ABC) (5-7). Differentiation of TOS 
from $A B C$ remains challenging even though the radiological and histopathological characteristics of TOS have been described by many researchers $(1,2,8)$. Because the radiological appearances of TOS are large, lucent lesions with fluid levels simulating those of $\mathrm{ABC}$, misdiagnoses and inaccurate diagnoses often lead to improper management, consequently affecting negatively the prognosis of the patients.

\section{Objectives}

The purpose of this study was to investigate and summarize the clinical, imaging and histopathological features of TOS and ABC in long tubular bones in our hospital in the recent decade for better evaluation and correct diagnosis of these two diseases.

\section{Patients and Methods}

This case-controlled retrospective study was approved by the Ethics Committee of our hospital. All patients or their legal guardians gave their written informed consent for clinical examination and treatment. The clinical data were reviewed at our hospital between 1990 and 2010 for patients with TOS or $A B C$ for comparison. The inclusion criteria were primary TOS or ABC confirmed by histopathology, presentation for the first time to the hospital without any treatment, and imaging examination of plain radiography, computed tomography (CT) or magnetic resonance imaging (MRI). Exclusion criteria were patients with TOS or ABC who had been treated and patients who had co-existence of other tumors. All patients had histopathological examinations. The imaging data, patients' age, sex, history of disease, location of lesions, symptoms, laboratory examination and pathology results were obtained through the PACS system (picture archiving and communication system) and HIS (hospital information system) system. The imaging signs were obtained with plain X-ray, CT, and MRI before treatment. Bone destruction, transitional band, periosteal reaction and soft tissue masses are assessed using plain films and CT, whereas soft tissue masses and fluid-fluid levels are evaluated with CT and MRI.

\subsection{Statistical Analysis}

IBM SPSS Statistics for Windows, version 24.0 (Armonk, NY: IBM Corp.) was used for analysis in this study. Measurement data were expressed as mean \pm SD (standard deviation), and the differences between groups were tested with the Mann-Whitney U test. The significant level was set at P $<0.05$.
Selection of paired $t$-test was not accurate because the two groups were independent and according to Kolmogorov-Smirnov test results, t-independent or MannWhitney test must have been applied.

\section{Results}

Seventeen patients with TOS in the long tubular bones confirmed by histopathology were retrieved including 10 males and seven females with an age range of 1 - 60 years (mean $25.4 \pm 8.7$ ) (Table 1). Seventeen patients with ABC were also retrieved for comparison including seven males and ten females with an age range of 3 - 43 years (mean 20.1 \pm 8.7 ) (Table 1). The general information regarding these patients is presented in Table 1 . All patients had plain radiography, twelve TOS and eleven ABC cases had both CT and MRI.

\begin{tabular}{|c|c|c|}
\hline & $\operatorname{TOS}(n=17)$ & $\operatorname{ABC}(\mathbf{n}=17)$ \\
\hline $\operatorname{Sex}(M / F)$ & $10 / 7$ & $7 / 10$ \\
\hline Onset age, $y$ & $25.4 \pm 8.7(1-60)$ & $20.1 \pm 8.7(3-43)$ \\
\hline Profession & Student (11) and farmer (6) & $\begin{array}{l}\text { Student (7), farmer (8), } \\
\text { and freelancer (2) }\end{array}$ \\
\hline Past history & $\begin{array}{l}\text { Healthy (15), hypertension } \\
\text { (1), smoking (1) and } \\
\text { alcohol abuse (1) }\end{array}$ & $\begin{array}{l}\text { Healthy (15), hypertension } \\
\text { (2) }\end{array}$ \\
\hline Lesion location & $\begin{array}{l}\text { Femur (11), humerus (2), } \\
\text { tibia (2) and ulna (2) }\end{array}$ & $\begin{array}{l}\text { Femur (7), tibia (6), } \\
\text { humerus (1), radius (1), } \\
\text { ulna (1) and fibula (1) }\end{array}$ \\
\hline Symptoms & $\begin{array}{l}\text { Local swelling (14) and } \\
\text { pathological fracture ( } 3 \text { ) } \\
\text { lasting } 3.0 \pm 1.5 \text { months } \\
\text { (range 1-5) }\end{array}$ & $\begin{array}{l}\text { Local pain or swelling (17) } \\
\text { and pathological fracture } \\
\text { (3) lasting } 9.8 \pm 10.1 \\
\text { months }(2 \mathrm{mo}-3 \mathrm{y})\end{array}$ \\
\hline Signs & $\begin{array}{l}\text { Soft tissue mass (9), high } \\
\text { skin temperature, (2) and } \\
\text { venous engorgement (2) }\end{array}$ & Hard apophysis (3) \\
\hline Laboratory & $\begin{array}{l}\text { Rise in serum alkaline } \\
\text { phosphatase (3) }\end{array}$ & $\begin{array}{l}\text { Rise in serum alkaline } \\
\text { phosphatase (1) }\end{array}$ \\
\hline Treatment & $\begin{array}{l}\text { Local resection }+ \text { artificial } \\
\text { joint replacement }(4) \text { and } \\
\text { local resection }+ \\
\text { chemotherapy (13) }\end{array}$ & $\begin{array}{l}\text { Curettage + bone grafting } \\
\text { + internal fixation (6), } \\
\text { curettage + bone cement } \\
\text { filling + internal fixation } \\
\text { (5), curettage + } \\
\text { inactivation + bone } \\
\text { grafting (3), curettage + } \\
\text { inactivation (1), curettage } \\
\text { + grafting (1) and } \\
\text { cephalectomy of ulna (1) }\end{array}$ \\
\hline
\end{tabular}

Abbreviations: ABC, aneurysmal bone cysts; F, female; M, male; SD, standard deviation; TOS, telangiectatic osteosarcoma.

${ }^{\mathrm{a}}$ Values are expressed as mean \pm SD or median (range).

No significant difference $(\mathrm{P}>0.05)$ existed at the age of disease onset between the two groups. However, the duration of symptom persistence was significantly $(\mathrm{P}=0.01)$ 
shorter in the TOS than in the ABC group before correct diagnosis (Table 1). Among 17 patients with TOS, the lesion was at the femur in eleven cases, tibia in two, humerus in two, and ulna in the remaining two. The presentation symptom was local swelling and pain in 14 cases and pathological fracture in the remaining three. The duration for symptoms was 1 - 5 months (mean $3.0 \pm 1.5$ ). A hard soft tissue mass was palpable in nine cases with ill-defined margins, local skin warmth in two, and varices in two. Laboratory tests were normal in nine cases, but increased alkaline phosphatase was found in three cases. Among the 17 patients with ABCs, the lesion was at the femur in seven cases, tibia in six, humerus in one, radius in one, fibula in one and ulna in the remaining one. The presenting symptoms were local pain or swelling lasting two months to three years (mean $9.8 \pm 10.1$ ) in all cases, with a hard soft tissue mass in one case and traumatic history in three. In the laboratory tests, the alkaline phosphatase was normal in all but one case.

\subsection{Plain Radiographs}

Bone destruction was classified into lytic and expansile destruction. In 17 TOS cases, a soft tissue mass was shown in 14 cases, lytic bone destruction in 13 (Figures 1 and 2 and Tables 2 and 3), ill-defined border with a wide transitional band in 12, periosteal reaction in eight, formation of the Codman triangle in five with radiating periosteal reaction in one case (Figure 3), pathological fracture in three and calcification in one. Three cases had slight expansion of the tumor, and five had marked expansile osteolytic changes. In 17 cases with $\mathrm{ABC}$, an ill-defined border was found in fifteen cases, lytic bone destruction in 17, marked expansile bone destruction in eleven, slightly expansile destruction in five, thinned sclerotic rim in twelve, eccentrically location in ten, coarse septations in eight (Figure 4), a soft tissue mass in three, pathological fracture in three, periosteal reaction in two and an ill-defined border with a wide transitional band in two.

\subsection{CT Imaging}

Expansive destruction, soft tissue masses, pathological fracture and transitional band were detected more clearly in CT than in plain radiography (Tables 2 and 3). In TOS, multiple cysts were shown in twelve cases, and fluid-fluid levels in three. In $\mathrm{ABC}$, multiple cysts were present in eleven cases while fluid-fluid levels in four. Irregular rims were seen in nine cases, with slight invasion of the endosteum. In two cases, the endosteum was apparently invaded, with an ill-defined border and periosteal reaction in one, similar to that of eosinophilic granuloma.

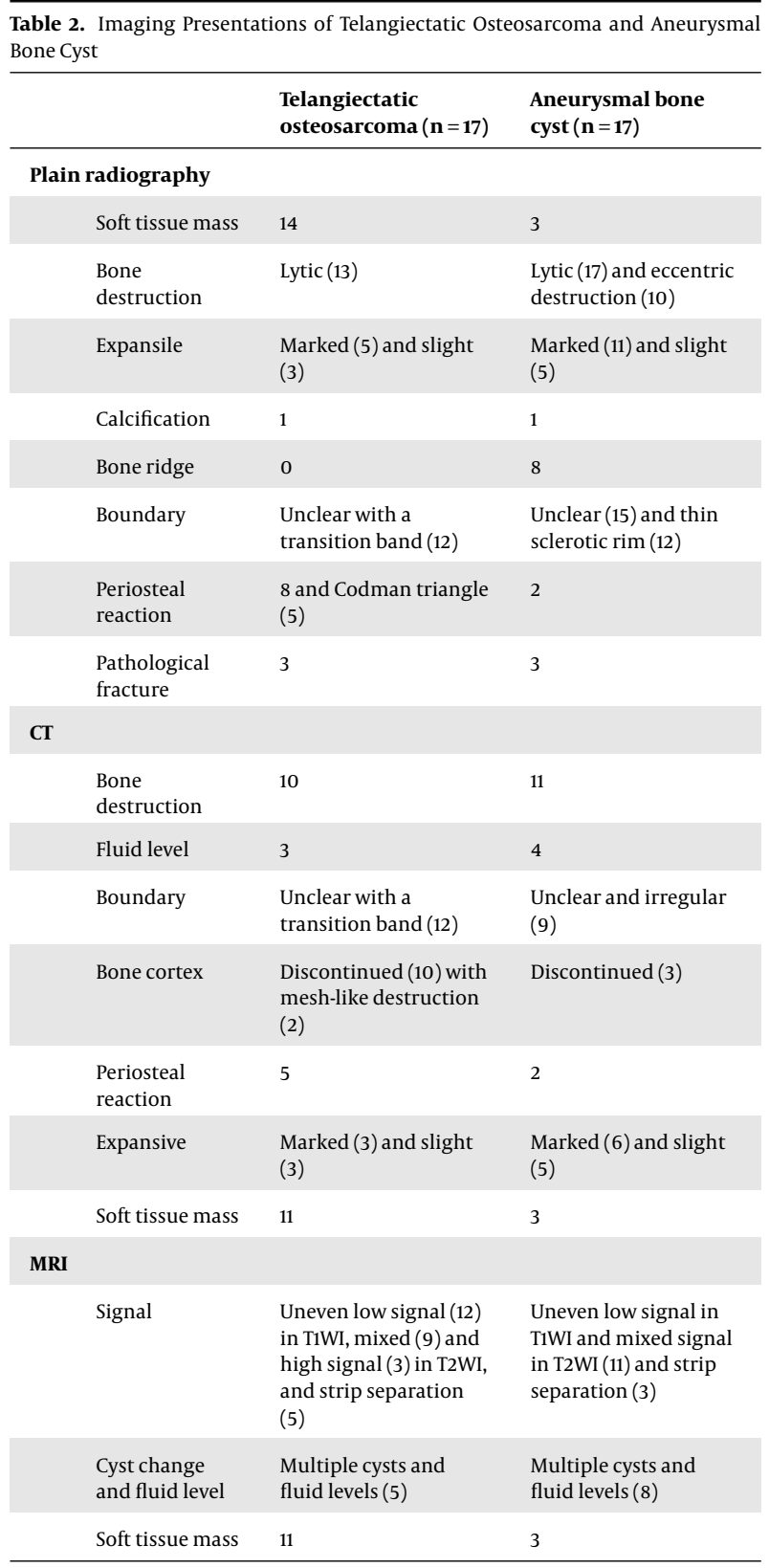

Abbreviations: CT, computed tomography; MRI, magnetic resonance imaging.

\subsection{MRI Presentations}

In TOS, low and medium signal was present in the T1weighted imaging(T1WI), whereas mixed (9/12) or high signal (3/12) was present in the T2 WI with inhomogeneous signal (Tables 2 and 3). Most tumors had beehive like changes, and four cases had multiple cysts and fluid-fluid levels in the bone destruction and soft tissues (Figure 1). The cysts had long T1 and long T2 signal with isointense signal in the septations (5/12). Fat inhibition sequence showed clear cys- 

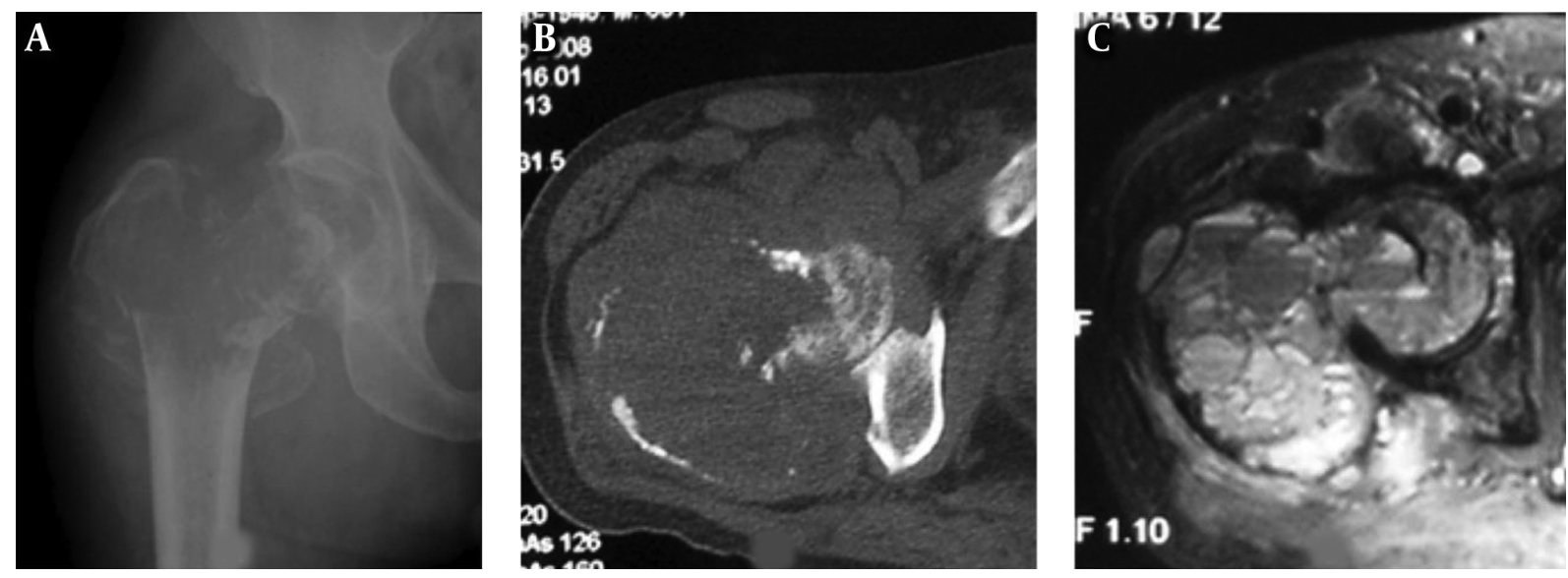

Figure 1. A 60-year-old man had an intertrochanteric telangiectatic osteosarcoma accompanied with pathological fracture. A and B, A lytic destruction lesion is shown with interrupted cortex, ill-defined margins, wide transitional band but no tumorous bone inside the lesion; C, The T2-weighted imaging (T2WI) of magnetic resonance imaging showed multiple fluid-fluid levels.
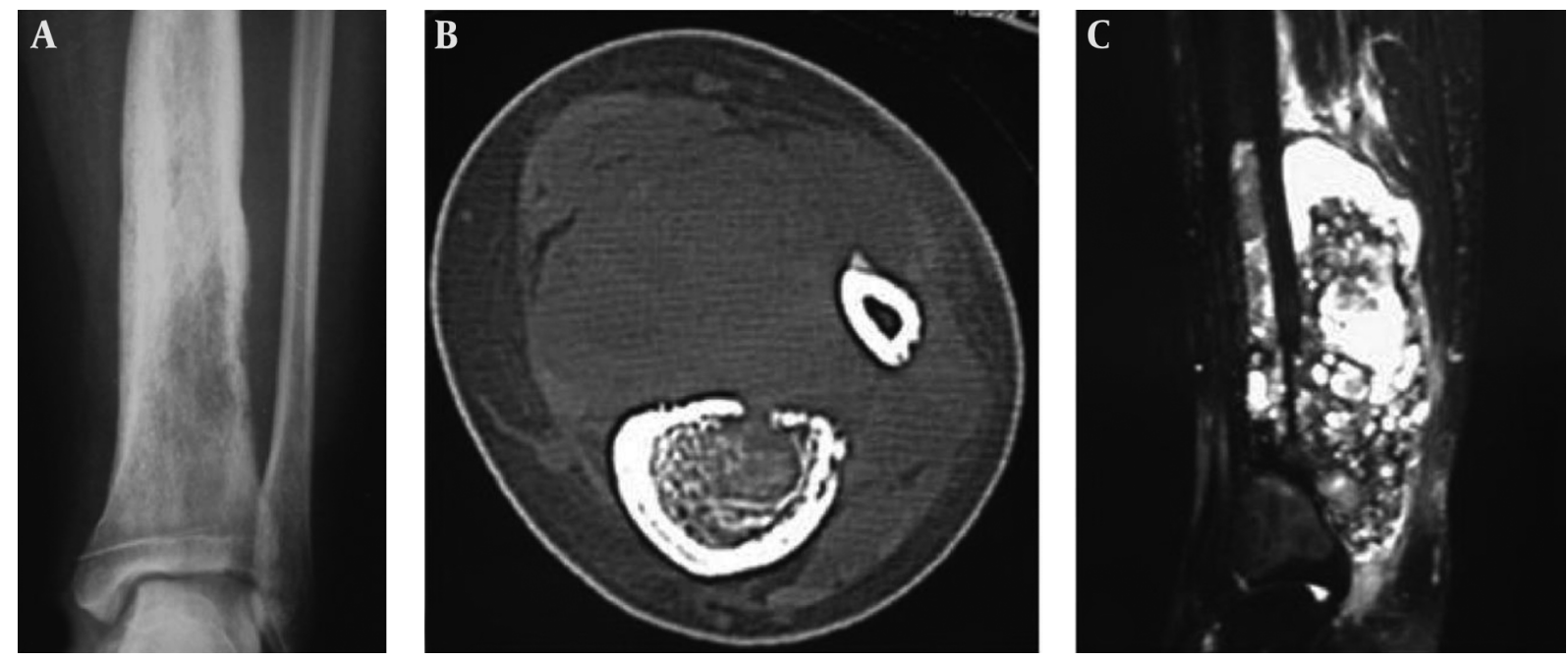

Figure 2. A 22-year-old girl who had a telangiectatic osteosarcoma in the left tibia. A and B, A lytic lesion with adjacent sclerotic changes is shown with ill-defined margins. The cortex is eroded and irregularly thickened; C, Fat suppression sequence of T2-weighted imaging (T2WI) of magnetic resonance imaging revealed the bone lesion and mixed hyperintense signal in the soft tissue mass.

tic changes. In T1WI, the fluid-fluid levels had hypointense signal in the upper level but isointense signal in the lower level, whereas in T2 WI, hyperintense and isointense signals (5/12) were present in the lower level. One case with TOS had MRI enhancement with inhomogeneous enhancement in the tumor tissues but no enhancement in the cystic area.

In $\mathrm{ABC}$, low complete or incomplete signal was demonstrated in all lesions with a higher incidence of fluid-fluid levels (8/11). The lesion was of low and medium inhomogeneous signal in the T1 WI but mixed signal in T2weighted imaging(T2WI)(6/11) including hyperintense sig- nal in the upper part but mixed signal in the lower part in four cases or hyperintense signal (1/11) in T2 WI. In one case with pathological fracture, hypointense signal was demonstrated near the fracture line in T1 and T2 WI.

\subsection{Comparison of the Imaging Features}

Patients with TOS had a significantly $(\mathrm{P}<0.05)$ higher prevalence of periosteal reaction, soft tissue masses, wide transitional band and ill-defined margins than those with $\mathrm{ABC}$, whereas patients with $\mathrm{ABC}$ had a significantly $(\mathrm{P}<$ 0.05) higher incidence of thinned sclerotic rims. 


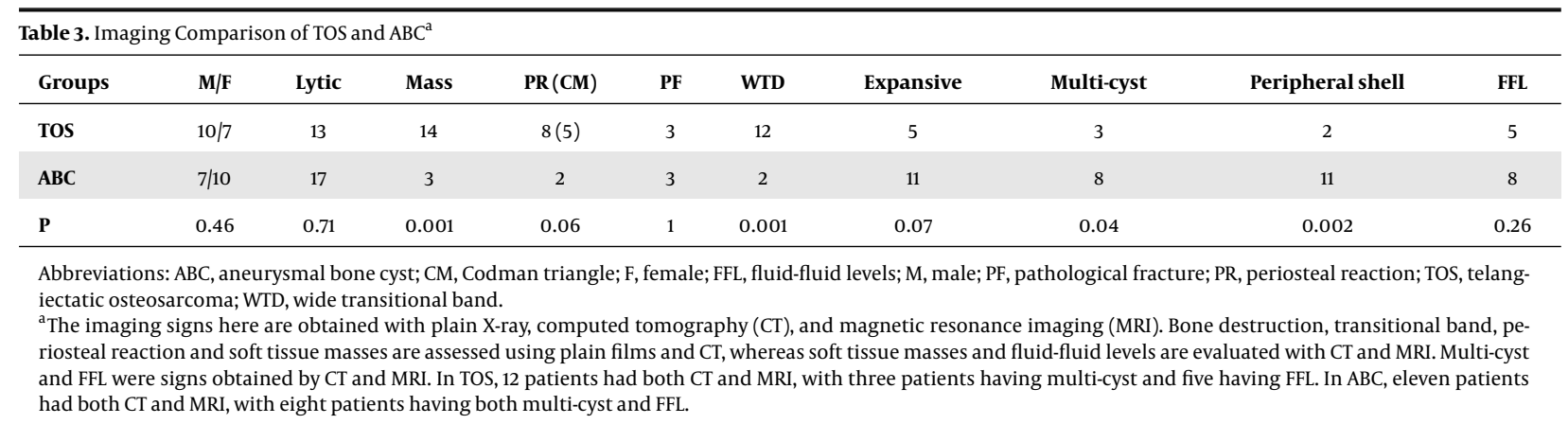
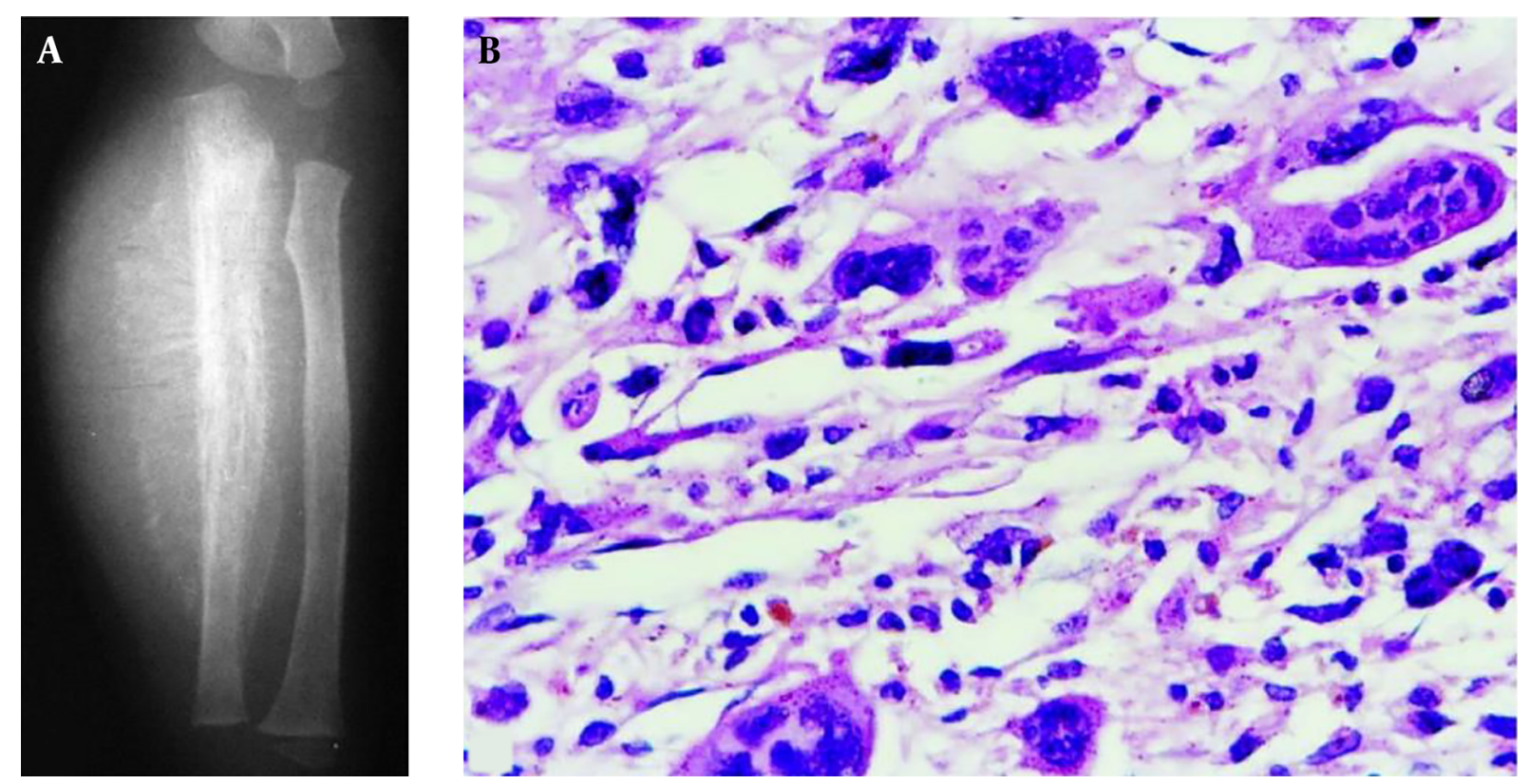

Figure 3. A 1.5-year-old boy had a telangiectatic osteosarcoma in the ulna. A, The lesion is lytic with radiating periosteal reaction, a soft tissue mass and Codman triangle similar to conventional osteosarcoma; B, The histopathological staining shows heterocysts and atypical karyodieresis in the septa (H\&E $400 \times)$.

\subsection{Histopathology}

All the cases with TOS and ABC were confirmed with histopathology.

In TOS, preoperative biopsy was performed in twelve cases with a feeling of emptiness and expulsion of a large amount of blood. Among these twelve cases, eight were definitively diagnosed as a malignant tumor while four had no tumor tissues in the biopsy. In 17 cases with TOS, postoperative gross specimen demonstrated local expansion, multiple cysts at the cross section and rufous blood clots in the cysts in thirteen cases (13/17). The tumor tissues invaded and even broke through the cortex, which became thinned and interrupted with ill-defined borders. In low power microscopy, big cysts with blood or clots existed in the tumors, similar to that of ABC. In high power microscopy, the TOS tumor cells in the interval and septa- tions were of high anaplasia, polymorphy and atypia (Figure 3), with a lot of mitotic imaging, pathological mitotic imaging, tumorous osteoid tissue and sparsely distributed polykaryocytes. In four cases, tumorous bone tissue was formed.

In $\mathrm{ABC}$, preoperative biopsy was performed in thirteen cases, with bloody cysts containing hyperplastic fibroblasts in the septations that had sparsely distributed osteoclast-like polykaryocytes and woven bone covered with osteoblasts. The fibroblasts and osteoblasts had not atypia or pathological mitotic imaging.

\section{Discussion}

As a rare subtype of osteosarcoma, TOS was firstly described by Paget (2), and Gaylord (9) thought that these le- 

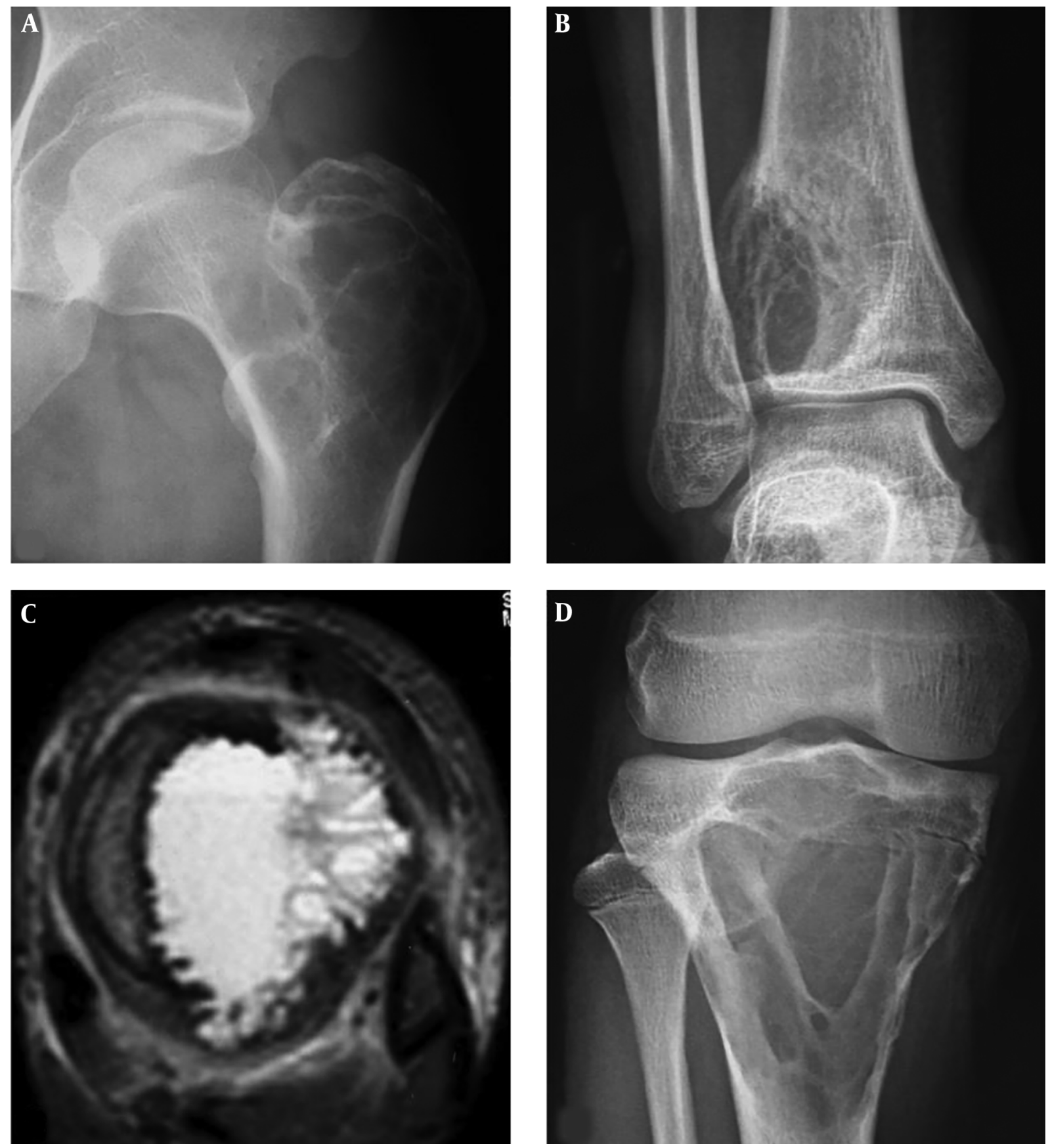

Figure 4. A, A 22-year-old man with an intertrochanteric aneurysmal bone cyst (ABC) with a bone destruction lesion and multiple cysts, clear margins and coarse septa; $B$ and C, A 15-year-old girl with a tibial ABC; B, A Cystic lytic destructive lesion is seen in the distal tibia with clear margins and coarse septations; C, T2-weighted imaging (T2WI) magnetic resonance imaging demonstrated fluid-fluid levels; D, A 17-year-old boy with a tibial ABC and a cystic lytic lesion, thinned cortex, coarse septa and clear margins.

sions were malignant aneurysms of the bone in 1903. However, it was Ewing who was the first to consider TOS as a variant of osteogenic sarcoma (2). In 1976, Matsuno et al. (10) reported 25 cases of TOS and proposed the standard for diagnosis. TOS is characterized by multiple, aneurysmallydilated, blood-filled cavities with high-grade sarcomatous cells at the peripheral rim and within the septa (1-4). Similar to conventional osteosarcoma, TOS has the same distri- 
bution in race, clinical symptoms and location in the bone (3). ABCs are benign intraosseous solitary tumors consisting of large thin-walled cavities, which are filled with blood when examined macroscopically $(11,12)$. ABCs are rare bony tumors constituting only $1 \%-2 \%$ of all bone tumors (12, 13). ABCs were considered initially to be pseudotumors, but identification of fusion genes indicated that primary ABCs are true bone neoplasms (14). Even though the clinical, pathological and imaging characteristics of TOS have been sufficiently described in the literature $(1,2,8)$, reaching a correct diagnosis remains challenging, and malignant TOS can be readily misdiagnosed as benign ABC.

In our study, TOS was more prevalent in males than in females (10:17 or 58.8\%) with a mean onset age of 25.4 years consistent with the literature, with the percentage of male prevalence of $54 \%$ - $67 \%$ in previous studies $(1,2,4)$ and $58.8 \%$ in our study. Pathological fracture occurred in three of seventeen patients (17.6\%) in our study but was reported as 33\% in the literature (4), which is much higher than that for conventional osteosarcoma $(6 \%-13 \%)(15,16)$. This high incidence of pathological fracture may be caused by massive bone destruction in TOS (4). TOS mainly occurs at the metaphyses of long tubular bones, especially in the lower extremities. The alkaline phosphatase was mostly normal, which is quite different from conventional osteosarcoma, probably due to inactivity of pathological ossification in the tumor. $\mathrm{ABC}$ has a higher incidence than TOS and can be divided into primary and secondary subtypes. The age is younger for $\mathrm{ABC}$ than for TOS, and $\mathrm{ABC}$ is more prevalent in females than in males. Various theories have been suggested for the origin of $\mathrm{ABC}$ including arteriovenous shunts, traumatic bleeding and bleeding from a prior bony lesion; however, the pathogenesis remains to be determined (17-19). Trauma is a frequent inducement factor probably leading to venous occlusion or arteriovenous shunts to change the local hemodynamic status.

In imaging, TOS is characterized by bone destruction with malignant features including periosteal reaction, Codman triangle, soft tissue masses, ill-defined margins, wide transitional band, pathological fracture, and tumor ossification/calcification, which indicate malignancy of tumors. ABCs are locally destructive and expansile cystic lesions with coarse bony septa, narrow transitional band and sclerotic rim, which are characteristics of benign tumors. Multiple cysts and fluid-fluid levels can both be seen in these two diseases. However, in TOS, the fluid-fluid levels had hypointense signal in the upper level and isointense signal in the lower level in T1WI, but hyperintense and isointense signal (5/12) in the lower level in T2 WI. The fluidfluid levels indicate bleeding inside the lesion and are nonspecific signs (7). CT or MRI enhancement in TOS showed thick cystic wall, septa and strong nodular enhancement.
In $\mathrm{ABC}$, the enhancement is not as strong as in TOS. In $\mathrm{ABC}$, the complete or incomplete low signal rim in all lesions in MRI may be a fibrous membrane envelope, sclerotic rim or bone shell, with a higher incidence than in TOS, which is an imaging feature different from TOS. The features helpful for differentiation of TOS from $A B C$ are periosteal reaction, soft tissue masses, wide transitional band and high incidences of ill-defined borders, and a sclerotic rim has a higher incidence in ABC than in TOS. The long tubular bones in limbs do not overlap, and plain films and CT have the same diagnostic effect in evaluating bone destruction, edges of destruction and periosteal reaction in long tubular bones. However, CT can better show details of bone destruction, soft tissue masses and lesion edges. MRI is better in demonstrating lesion range and fluid-fluid levels. Combination of the three imaging modalities helps making a correct diagnosis.

TOS and $\mathrm{ABC}$ have similar pathological features of multiple cysts filled with blood, septa and colored band in low power microscopy. In high power microscopy, the blood cyst septa and interval in TOS had tumor cells of high anaplasia, polymorphy and atypia with pathological mitotic imaging; whereas, the cells in the cystic septa had neither cellular atypia nor pathological mitotic imaging. In one case with TOS, radiating tumor bone occurred similar to conventional osteosarcoma, which is very rare and should be combined with clinical, imaging and pathological data for correct diagnosis. Aspiration biopsy should not be performed for pathological examination because the possibility to obtain malignant septa tumor cells is very low, and open biopsy should be performed especially when aspiration biopsy cannot make a correct diagnosis.

In conclusion, clinical, imaging and pathological characteristics should be combined to make a correct differential diagnosis between telangiectatic osteosarcoma and aneurysmal bone cyst.

\section{Footnotes}

Authors' Contributions: Study design: Yun-Heng Shi, Wen-Juan Wu, and Bu-Lang Gao. Data collection and analysis: Yun-Heng Shi, Shu-Man Han, Tao Sun, and Wen-Juan Wu. Supervision: Bao-Hai Yu and Hui-Zhao Wu. Statistics: Yun-Heng Shi and Hui-Zhao Wu. Manuscript writing: YunHeng Shi. Manuscript revision: Bu-Lang Gao. Approval of the manuscript: all authors.

Conflict of Interests: The authors declare that they have no conflict of interest.

Ethical Approval: This study was approved by the Ethics Committee of the Third Hospital of Hebei Medical University. The ethical approval code is $201700540 \mathrm{TH}$. 
Funding/Support: No funding was received for this study. Informed Consent: Written informed consent was obtained from all patients or their legal guardians.

\section{References}

1. Gao ZH, Yin JQ, Liu DW, Meng QF, Li JP. Preoperative easily misdiagnosed telangiectatic osteosarcoma: Clinical-radiologicpathologic correlations. Cancer Imaging. 2013;13(4):520-6. doi: 10.1102/1470-7330.2013.0042. [PubMed: 24334494]. [PubMed Central: PMC3864225].

2. Sangle NA, Layfield LJ. Telangiectatic osteosarcoma. Arch Pathol Lab Med. 2012;136(5):572-6. doi: 10.5858/arpa.2011-0204-RS. [PubMed: 22540307].

3. Skiadas V, Koutoulidis V, Koureas A, Moulopoulos L, Gouliamos A. Plain X-ray, computed tomography and magnetic resonance imaging findings of telangiectatic osteosarcoma: A case report. Cases J. 2009;2:7833. doi: 10.4076/1757-1626-2-7833. [PubMed: 19918488]. [PubMed Central: PMC2769378].

4. Yin JQ, Fu YW, Xie XB, Cheng XY, Yang XY, Liu WH, et al. Telangiectatic osteosarcoma: Outcome analyses and a diagnostic model for differentiation from aneurysmal bone cyst. J Bone Oncol. 2018;11:10-6. doi: 10.1016/j.jbo.2017.11.003. [PubMed: 29892520]. [PubMed Central: PMC5993956].

5. Liu JJ, Liu S, Wang JG, Zhu W, Hua YQ, Sun W, et al. Telangiectatic osteosarcoma: A review of literature. Onco Targets Ther. 2013;6:593602. doi: 10.2147/OTT.S41351. [PubMed: 23745051]. [PubMed Central: PMC3671797].

6. Menendez LR, Bacon W, Kempf RA, Moore TM. Fat embolism syndrome complicating intraarterial chemotherapy with cis-platinum. Clin Orthop Relat Res. 1990;(254):294-7. [PubMed: 2323145].

7. Weiss A, Khoury JD, Hoffer FA, Wu J, Billups CA, Heck RK, et al. Telangiectatic osteosarcoma: The St. Jude Children's Research Hospital's experience. Cancer. 2007;109(8):1627-37. doi: 10.1002/cncr.22574. [PubMed: 17351949].

8. Murphey MD, wan Jaovisidha S, Temple HT, Gannon FH, Jelinek JS, Malawer MM. Telangiectatic osteosarcoma: Radiologic-pathologic comparison. Radiology. 2003;229(2):545-53. doi: 10.1148/radiol.2292021130. [PubMed: 14512511].

9. Gaylord HR. III. on the pathology of so-called bone aneurisms. Ann Surg. 1903;37(6):834-47. [PubMed: 17861305]. [PubMed Central: PMC1431073].
10. Matsuno T, Unni KK, McLeod RA, Dahlin DC. Telangiectatic osteogenic sarcoma. Cancer. 1976;38(6):2538-47. doi: 10.1002/10970142(197612)38:6<2538::Aid-cncr2820380643>3.0.Co;2-1.

11. Ruiter DJ, Cornelisse CJ, van Rijssel TG, van der Velde EA. Aneurysmal bone cyst and telangiectatic osteosarcoma. A histopathological and morphometric study. Virchows Arch A Pathol Anat Histol. 1977;373(4):311-25. doi: 10.1007/bfo0432531. [PubMed: 140511].

12. Sasaki H, Nagano S, Shimada H, Yokouchi M, Setoguchi T, Ishidou Y, et al. Diagnosing and discriminating between primary and secondary aneurysmal bone cysts. Oncol Lett. 2017;13(4):22906. doi: 10.3892/ol.2017.5682. [PubMed: 28454393]. [PubMed Central: PMC5403183].

13. Boubbou M, Atarraf K, Chater L, Afifi A, Tizniti S. Aneurysmal bone cyst primary-about eight pediatric cases: Radiological aspects and review of the literature. Pan Afr Med J. 2013;15:111. doi 10.11604/pamj.2013.15.111.2117. [PubMed: 24244797]. [PubMed Central: PMC3828064].

14. Oliveira AM, Perez-Atayde AR, Inwards CY, Medeiros F, Derr V, Hsi BL, et al. USP6 and CDH11 oncogenes identify the neoplastic cell in primary aneurysmal bone cysts and are absent in so-called secondary aneurysmal bone cysts. Am J Pathol. 2004;165(5):1773-80. doi 10.1016/S0002-9440(10)63432-3. [PubMed: 15509545]. [PubMed Central: PMC3278819].

15. Scully SP, Ghert MA, Zurakowski D, Thompson RC, Gebhardt MC Pathologic fracture in osteosarcoma: Prognostic importance and treatment implications. J Bone Joint Surg Am. 2002;84(1):49-57. [PubMed: 11792779].

16. Scully SP, Temple HT, O'Keefe RJ, Mankin HJ, Gebhardt M. The surgical treatment of patients with osteosarcoma who sustain a pathologic fracture. Clin Orthop Relat Res. 1996;(324):227-32. doi: 10.1097/00003086-199603000-00028. [PubMed: 8595761].

17. Bollini G, Jouve JL, Cottalorda J, Petit P, Panuel M, Jacquemier M. Aneurysmal bone cyst in children: Analysis of twenty-seven patients. J Pediatr Orthop B. 1998;7(4):274-85. doi: 10.1097/01202412-19981000000005. [PubMed: 9810526$]$

18. Campanacci M, Capanna R, Picci P. Unicameral and aneurysmal bone cysts. Clin Orthop Relat Res. 1986;(204):25-36. [PubMed: 3956013].

19. Gibbs CJ, Hefele MC, Peabody TD, Montag AG, Aithal V, Simon MA Aneurysmal bone cyst of the extremities. Factors related to local recurrence after curettage with a high-speed burr. J Bone Joint Surg Am. 1999;81(12):1671-8. doi: 10.2106/00004623-199912000-00003. [PubMed: 10608377]. 